\title{
Publisher's Note: Orthogonal tight-binding model for the thermal conductivity of Si [Phys. Rev. B 93, 155203 (2016)]
}

Ankita Katre and Georg K. H. Madsen

(Received 18 April 2016; published 4 May 2016)

DOI: 10.1103/PhysRevB.93.199901

This paper was published online on 11 April 2016 with an error in the text on page 1 and on page 6. On page 1, left-hand column, the tenth line of the second paragraph should read as "...theory (DFT) based thermal conductivity calculations...." On page 6, right-hand column, the fifth line of the second paragraph should read as "energies for each structure at $\pm 15 \%$ volume range). The rest...." The paper has been corrected as of 2 May 2016. The text is correct in the printed version of the journal. 